\title{
Luteolin exerts an anticancer effect on gastric cancer cells through multiple signaling pathways and regulating miRNAs
}

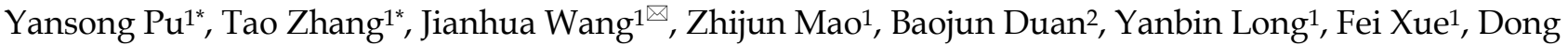 \\ $\mathrm{Liu}^{1}$, Sida Liu ${ }^{1}$, Zengzhan Gao ${ }^{1}$ \\ 1. The Second Department of General Surgery, Shaanxi Provincial People's Hospital, Xi'an, Shaanxi 710068, P.R. China. \\ 2. Department of Oncology, Shaanxi Provincial People's Hospital, Xi'an, Shaanxi 710068, P.R. China. \\ *Dr Yansong Pu and Dr Tao Zhang contributed equally to this work. \\ $\triangle$ Corresponding author: Jianhua Wang, MD, PhD, The Second Department of General Surgery, Shaanxi Provincial People's Hospital, Xi'an, Shaanxi 710068, \\ P.R. China. \\ (c) Ivyspring International Publisher. This is an open access article distributed under the terms of the Creative Commons Attribution (CC BY-NC) license \\ (https://creativecommons.org/licenses/by-nc/4.0/). See http://ivyspring.com/terms for full terms and conditions.
}

Received: 2018.05.09; Accepted: 2018.08.02; Published: 2018.09.08

\begin{abstract}
Accumulating studies confirmed that luteolin, a common dietary flavonoid which is widely distributed in plants and has diverse beneficial biological function, including anti-oxidant, anti-inflammation and anticancer properties. However, the detail mechanisms of luteolin on GC are poorly understood. Here, we investigated the anticancer effect of luteolin in GC cells in vitro and in vivo. Luteolin reduced the cell viability in a time and dose-dependent manner. Luteolin significantly inhibited cell cycle progress, colony formation, proliferation, migration, invasion and promoted apoptosis in vitro and in vivo. Luteolin also regulated these biological effects associated regulators. Mechanically, luteolin treatment regulated Notch 1, PI3K, AKT, mTOR, ERK, STAT3 and P38 signaling pathways and modulated a series of miRNAs expression. These findings provide novel insight into the molecular function of luteolin which suggest its potential as a therapeutic agent for human GC.
\end{abstract}

Key words: Luteolin, gastric cancer, EMT, prognosis, metastasis

\section{Introduction}

Gastric cancer (GC) is one of the most common gastrointestinal malignancies and the leading cause of cancer-related mortality worldwide, especially in China [1-3]. Despite the development of endoscopy examination significantly increased the rate of earlier diagnosis of GC and timely surgical resection gives a favorable outcome, the long-term survival is still not satisfactory due to the high rate of metastasis, recurrence drug-resistance and no effective approaches to reverse advanced GC [4-6]. Therefore, it's necessary to develop novel therapeutic reagents to treat GC. Numerus studies reported that the long-term consumption of food in fruits and vegetables reduces the risk of cancer [7, 8]. Thus, exploiting natural phytochemicals for developing anticancer agents which are more effective and less toxic remains an important priority.
Luteolin $\left(3^{\prime}, 4^{\prime}, 5,7\right.$-tetrahydroxyflavone), is a natural flavonoid which widely exist at high concentration in fruits and vegetables [9]. Recent studies confirmed that luteolin possessed various pharmacological effects including anticancer, antioxidant, anti-inflammatory, immune regulation and cardio protective [10-12]. Increasing evidence demonstrated that luteolin exerted in anti-tumor activities in several cancers [13-15]. Luteolin induced growth inhibition and apoptosis of human esophageal squamous carcinoma cell line Eca109 cells in vitro [16]. Luteolin exerts pro-apoptotic effect and anti-migration effects on A549 lung adenocarcinoma cells through the activation of MEK/ERK signaling pathway [17]. Luteolin inhibits migration of human glioblastoma U87 MG and T98G cells through downregulation of Cdc42 expression and PI3K/AKT activity [18]. Luteolin enhances paclitaxel-induced 
apoptosis in human breast cancer MDA-MB-231 cells by blocking STAT3 [19]. Luteolin inhibits cell proliferation and induces cell apoptosis via down-regulation of mitochondrial membrane potential in esophageal carcinoma cells EC1 and KYSE450 [20]. These studies confirmed the anti-cancer activity in cancers. Luteolin also can regulated miRNAs expression in different cancer to affect the cancer progression. However, the underlying molecular mechanism and effects of luteolin on human gastric cancer needs to be validated.

In this study, we evaluated the function of luteolin in regulating gastric cancer cell proliferation, migration and invasion. Moreover, we investigated luteolin intervention modulated multiple signaling pathways and miRNAs expression and illustrated the underlying mechanism.

\section{Materials and methods}

\section{Cell culture}

The human GC cell lines (MKN45 and BGC823) were maintained in RPMI1640 (Invitrogen, Carlsbad, USA) added with $10 \%$ fetal bovine serum (FBS; HyClone, Logan, UT, USA) and 1\% penicillin/ streptomycin (HyClone, Logan, UT, USA). All cell lines were incubated in a humidified atmosphere with $5 \% \mathrm{CO} 2$ at $37^{\circ} \mathrm{C}$.

\section{Immunohistochemistry (IHC) staining}

IHC assay was carried out on the $4 \mu \mathrm{m}$-thick formalin-fixed, paraffin-embedded section according to the protocol. The primary antibodies used in IHC staining were from Abcam (MA, USA). After incubated with the second antibodies, the slides were stained with diaminobenzidine for 3-10 $\mathrm{min}$ at room temperature and then observed by a light microscope.

\section{Quantitative reverse transcription-polymerase chain reaction ( $q R T-P C R$ )}

Total RNA of GC cells was extracted using TRIzol reagent according to the manufacturer's protocol. $1 \mu \mathrm{g}$ of total RNA was used to synthesize cDNA with the help of the PrimeScript RT reagent kit in reverse transcription reactions. $\mathrm{qPCR}$ was carried out on an ABI 7300 System using FastStart Universal SYBR Green Master with the primers. qPCR primers were ordered from Genecopoeia (Guangzhou, China). All experiments were repeated in triplicate.

\section{Western immunoblotting}

Cells and tissues were lysed on ice in RIPA lysis buffer according to manufacturer-provided instructions. The protein concentration was quantified with the BCA reagent (Rockford, IL, USA). Equal amounts of protein were divided to electrophoresis in
SDS-PAGE gel and then transferred to a nitrocellulose membrane (Millipore, MA, USA). After blocking with $5 \%$ non-fat milk for $1 \mathrm{~h}$, the membranes were incubated with antibodies respectively overnight at $4^{\circ} \mathrm{C}$, after washed 3 times with TBS $/ 0.1 \%$ Tween 20 , blots were incubated with HRP-conjugated secondary antibodies. Protein bands were visualized using an enhanced chemiluminescence kit (Amersham, Little Chalfont, UK).

\section{Cell migration and invasion assays}

Briefly speaking, $5.0 \times 10^{4}$ of GC cells were planted in basal medium without fetal bovine serum (FBS) in the upper chamber of Transwell chamber un-coated for migration assay or coated for invasion assay with $70 \mu \mathrm{L}$ Matrigel, while the lower chamber was filled with RPMI1640 medium with 20\% FBS. After 24 hours, GC cells traversed the filters were fixed with paraformaldehyde, stained with crystal violet after non-invasive cells were scrubbed off the upper surface of chamber. The traversed GC cells was photographed and counted under a microscopy.

\section{HCC xenograft assay}

All protocols involved in this experiment were approved by the Institutional Animal Care and Use Committee of Shaanxi Provincial People's Hospital of Xi'an Jiaotong University (Xi'an, China). Briefly, 4-6-week old nude mice were randomly divided into two groups equally. $1 \times 10^{7}$ MKN45 or BGC823 cells were resuspended in $100 \mu \mathrm{l}$ PBS and injected subcutaneously into the lateral flanks of each nude mouse. GC xenografts were examined by calipers weakly and the volume of xenografts was calculated using the following equation: Volume $=\mathrm{A} \times \mathrm{B}^{2} \times 0.25$ (A, length; B, width). Two weeks after injection, the animals were treated with PBS or luteolin (20 $\mathrm{mg} / \mathrm{Kg}$ ). After 2 weeks, all mice were sacrificed and the xenograft specimens were harvested.

\section{Statistical analysis}

All data were presented as means \pm SD and assessed by Student's t-tests or Mann-Whitney U test for comparison between groups. Statistical analysis was performed using GraphPad Prism 6 or SPSS 18.0. $P$ values less than 0.05 were considered statistically significant.

\section{Results}

\section{Luteolin inhibited cell growth of gastric cancer in vitro and in vivo}

Luteolin (Fig. 1A) is recognized as an anti-cancer drug candidate [21]. To validate the anti-tumor effect of luteolin on GC, we initially examined the cytotoxic effect of luteolin with different concentrations on GC 
cells (MKN45 and BGC823). The data showed that luteolin inhibited the growth of both MKN45 and BGC823 cells with a time- and dose- dependent effect relationship (Fig. 1B). As shown in Fig. 1B, the half-maximal inhibitory concentration (IC50) for both cells at $48 \mathrm{~h}$ or $72 \mathrm{~h}$ exposures was about $40 \mu \mathrm{M}$. Therefore, we choose 20 and $40 \mu \mathrm{M}$ for subsequent in vitro assays. The flow cytometry determined that luteolin treatment induced a significant G1 phase arrest and decreased the percentage of G2/M phase cells $(\mathrm{P}<0.05$, Fig. $1 \mathrm{C})$. Moreover, luteolin treatment increased the percentage of apoptosis in both MKN45 and BGC823 cells $(\mathrm{P}<0.05$, Fig. 1D). Furthermore, luteolin treatment significantly inhibited the colony formation $(\mathrm{P}<0.05$, Fig. $1 \mathrm{E})$. In addition, Edu assays confirmed that luteolin treatment remarkably inhibited the GC cell proliferation $(\mathrm{P}<0.05$, Fig. 1F).
These findings indicated that luteolin inhibited cell growth in gastric cancer.

To validate the antitumor effect of luteolin in vivo, we established the subcutaneous nude mice model with inoculation of MKN45 and BGC823 cells. The nude mice with tumors were injected 10 times intraperitoneally with PBS control or luteolin (20 $\mathrm{mg} / \mathrm{Kg}$ ). The data showed that luteolin treatment mice tumor volume and weight was less than the control group $(\mathrm{P}<0.05$, Fig. 2A-C). Moreover, we used IHC staining for Ki67, which was identified as a proliferation marker, to confirm that Ki67 expression was decreased in luteolin treatment mice $(\mathrm{P}<0.05$, Fig. 2D). In contrast, the TUNEL staining was increased in luteolin treatment mice $(\mathrm{P}<0.05$, Fig. 2E). These findings indicated that luteolin inhibited GC progression in vivo.

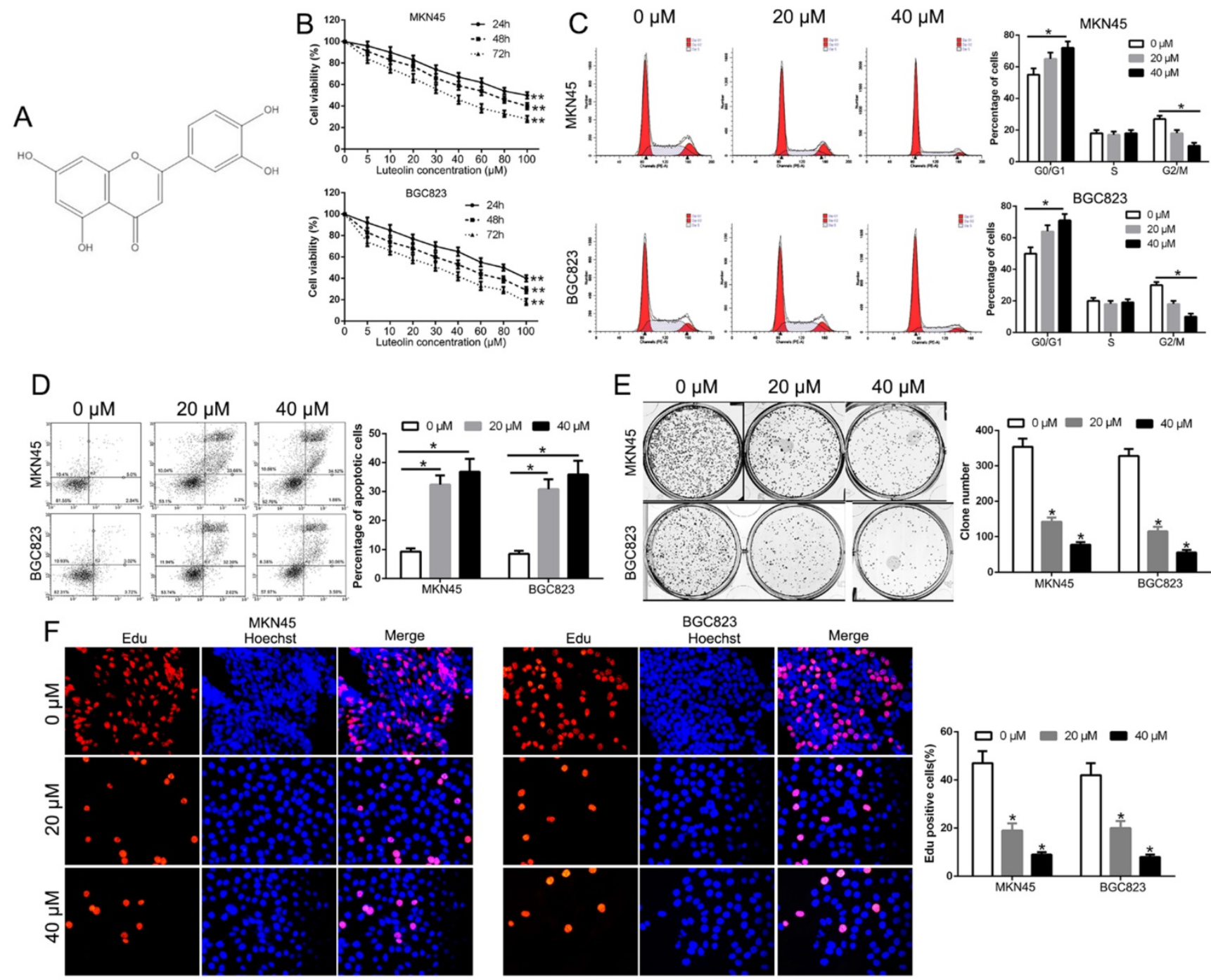

Figure 1. Effect of luteolin on cell viability, cell cycle, apoptosis, colony formation and proliferation. (A) The chemical structure of luteolin. (B) Cell survival analysis reveal that luteolin inhibits GC cell viability with a time- and dose-dependent relationship. Effects of luteolin on cell cycle (C), apoptosis (D), colony formation (E) and proliferation (F) on GC cells. $* \mathrm{P}<0.05, * * \mathrm{P}<0.01$. 

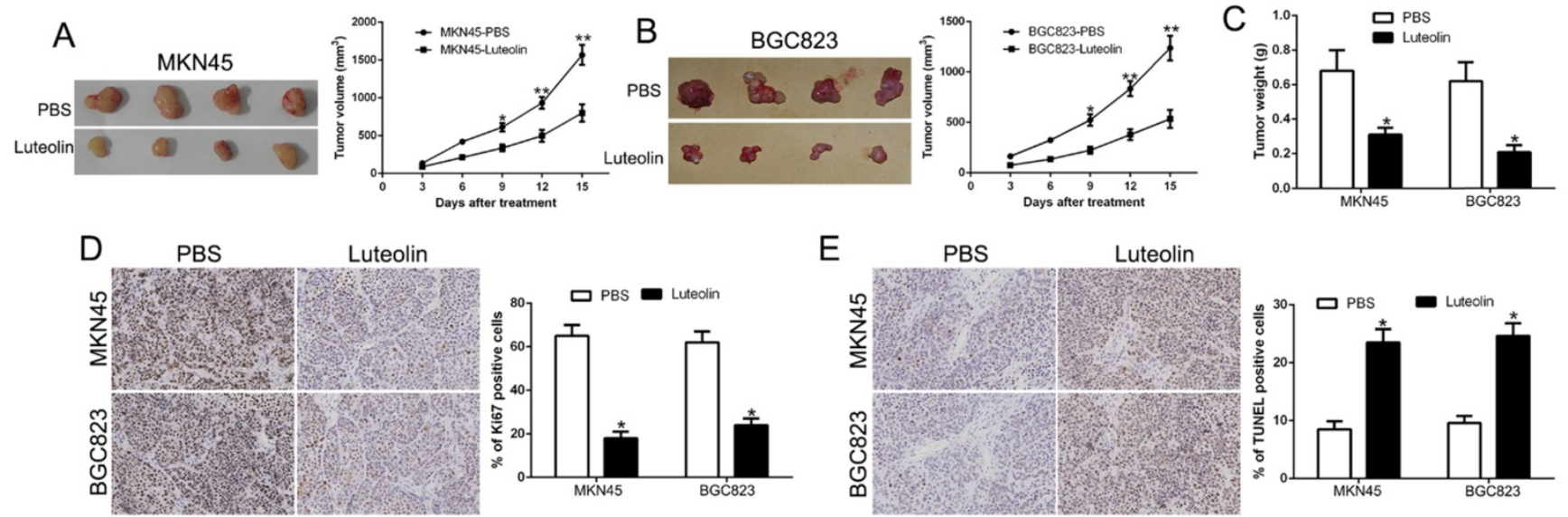

Figure 2. Effect of luteolin on tumor growth and metastasis in vivo. (A, B) Representative images of MKN45 and BGC823 xenograft treated with PBS or luteolin and tumor volumes were measured. (C) Average weights of xenograft tumors in nude mice. (D) Ki67 and (E) TUNEL staining of $x$ enograft tumors. $* P<0.05$, $* * P<0.01$.

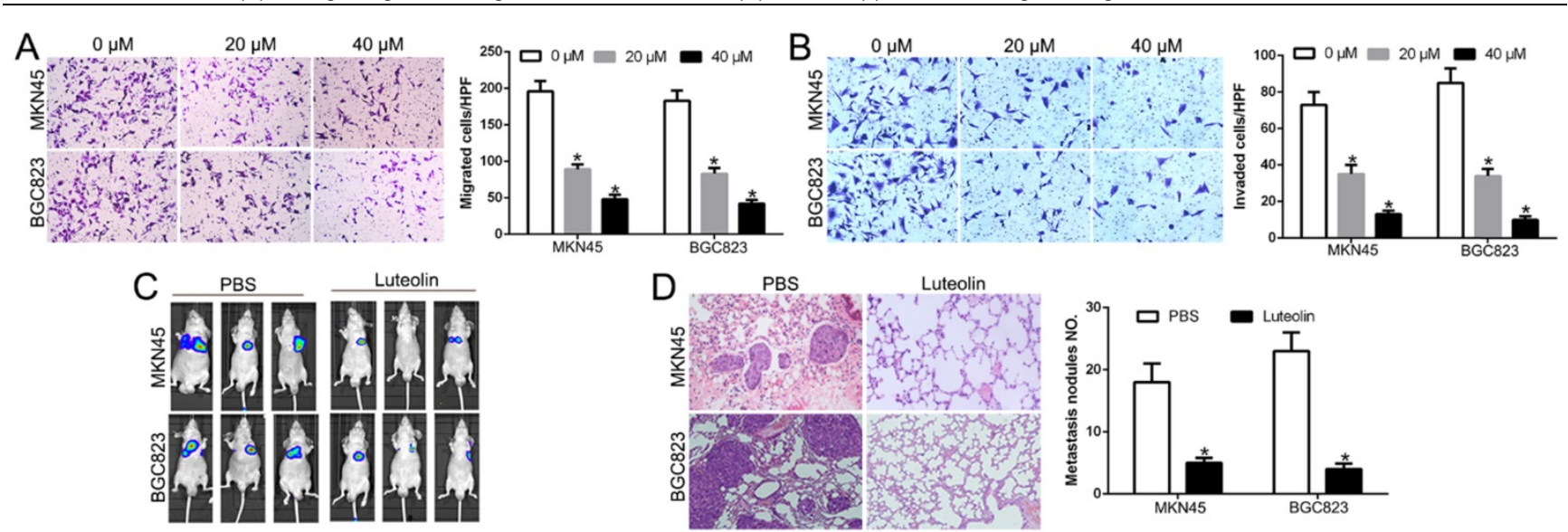

Figure 3. Luteolin suppressed GC cell migration and invasion in vitro and in vivo. Transwell un-coated (A) and coated- (B) Matrigel to measure the effects of luteolin on GC cells migration and invasion. (C) IVIS images show representative mice for PBS treated or Luteolin treated mice. (D) Lung colonization was analyzed by H\&E staining. *P $<0.05$.

\section{Luteolin suppressed GC cell migration and invasion in vitro and in vivo}

Metastasis plays a critical role in the prognosis of GC. To test the effect of luteolin in metastasis, we performed Transwell migration and invasion assays and found that luteolin inhibited GC cells migration and invasion in a dose-dependent manner $(\mathrm{P}<0.05$, Fig. 3A, B). To determine whether luteolin exerted its anticancer effects of gastric cancer lung metastasis in vivo, we injected GC cells with luciferase via tail vein. We measured the lung colonization of cancer cells by IVIS imaging. The data showed that luteolin treatment inhibited lung metastasis (Fig. 3C). HE staining further confirmed that luteolin blocked the lung colonization of GC cells in vivo $(\mathrm{P}<0.05$, Fig. 3D). These results suggest that luteolin inhibited GC cell migration in vitro and in vivo.

\section{Luteolin regulated the vital markers of biological function}

Above results showed that luteolin regulated GC cell cycle, apoptosis, colony formation, proliferation, migration and invasion function. To explore the molecular mechanisms by which luteolin regulated the biological function, we performed western blot to show that luteolin treatment inhibited Cyclin D1, Cyclin E, Bcl2, MMP2, MMP9, N-cadherin, Vimentin and promoted p21, Bax, E-cadherin expression (Fig. 4). These molecular are key regulators in previous biological function. These data indicated that these molecular participate in luteolin-induced biological effects.

\section{Luteolin suppressed the activation of multiple signaling pathways in GC}

To confirm the signaling pathway by which luteolin exerted its anticancer effects, we evaluated by western blot to measure diverse signaling pathways. The data showed that luteolin treatment reduced Notch1, p-PI3K, p-AKT, p-mTOR, p-ERK, p-STAT3 and increased p-P38 signaling (Fig. 5). These data suggest that these signaling pathways were all involved in luteolin-induced biological effects on GC cells. 
miRNA expression in luteolin-treated gastric cancer cells

Previous studies confirmed aberrant miRNAs regulated GC cell physiological and pathological process. We confirmed that miR-139, miR-34a, miR-422a, miR-107, which was confirmed as tumor suppressors [22-25], were significantly increased after luteolin treatment, while miR-21, miR-155, miR-224, miR-340, which was confirmed as oncogene [26-29], were remarkably decreased after luteolin treatment $(\mathrm{P}<0.05$, Fig. 6). These findings suggest that luteolin distinctively altered miRNA levels in gastric cancer cells.
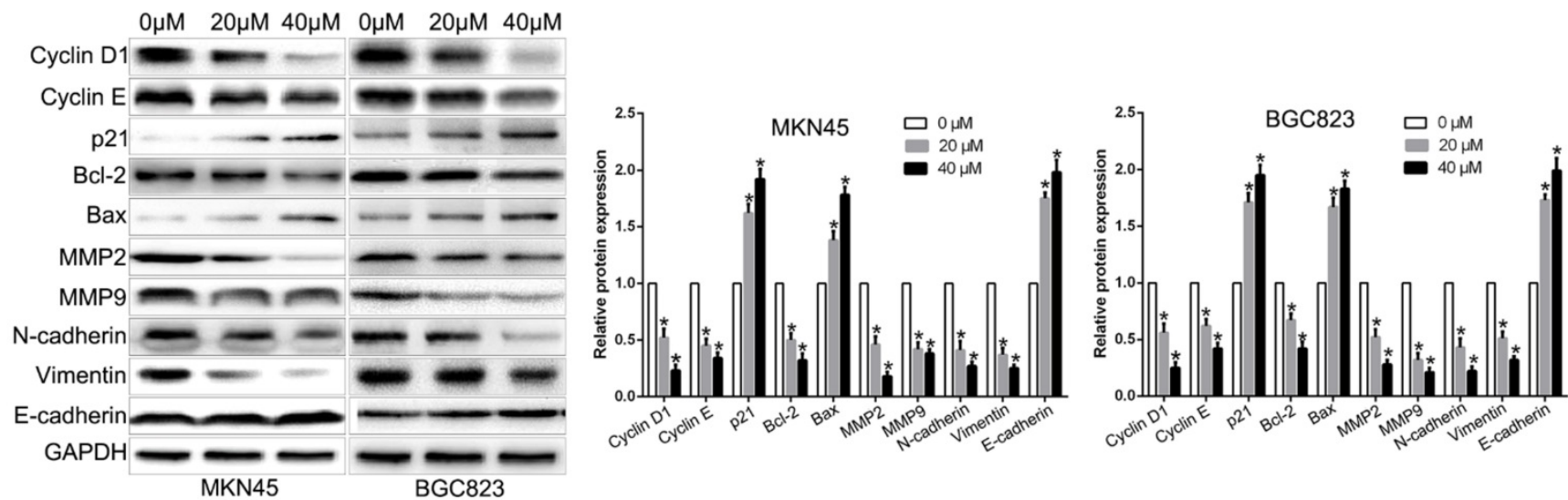

Figure 4. The cell cycle, apoptosis, proliferation, migration and invasion associated regulators expression levels after luteolin treatment.

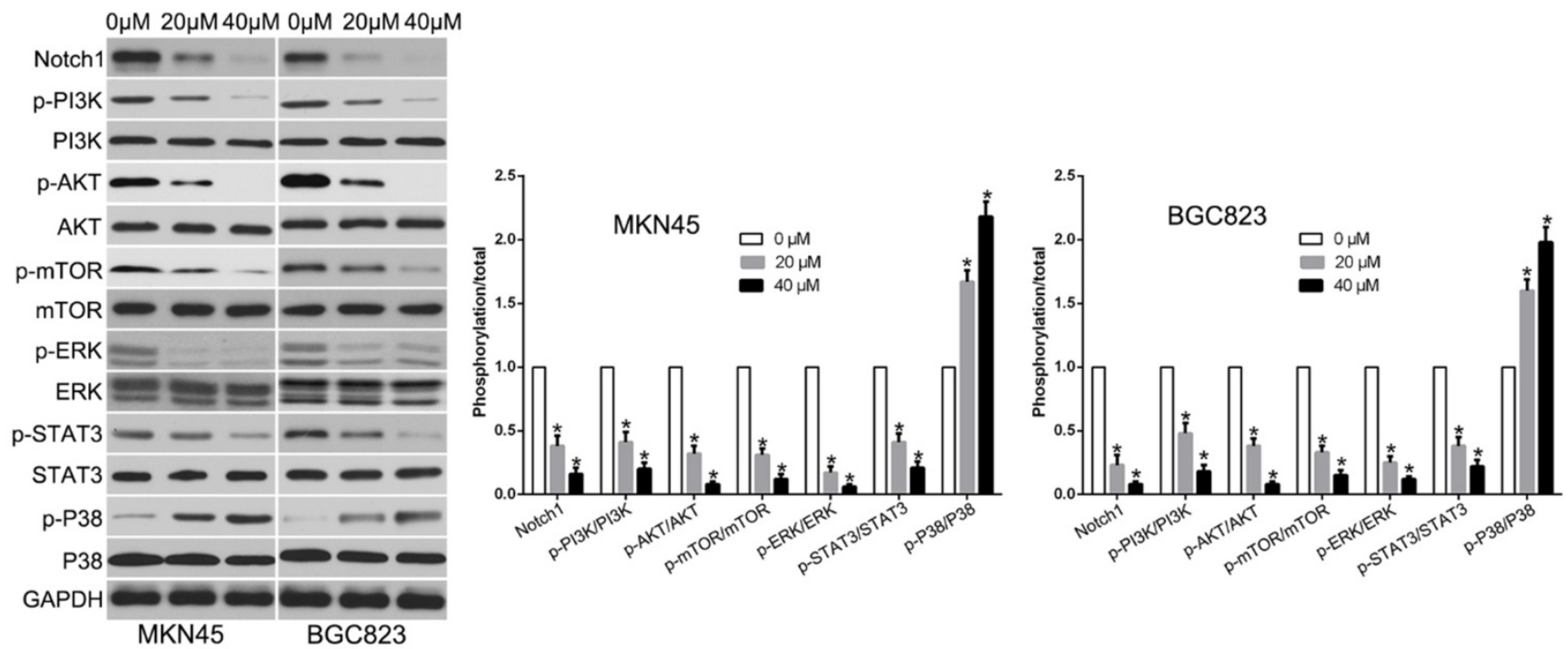

Figure 5. Luteolin treatment regulated multiple signaling pathways.
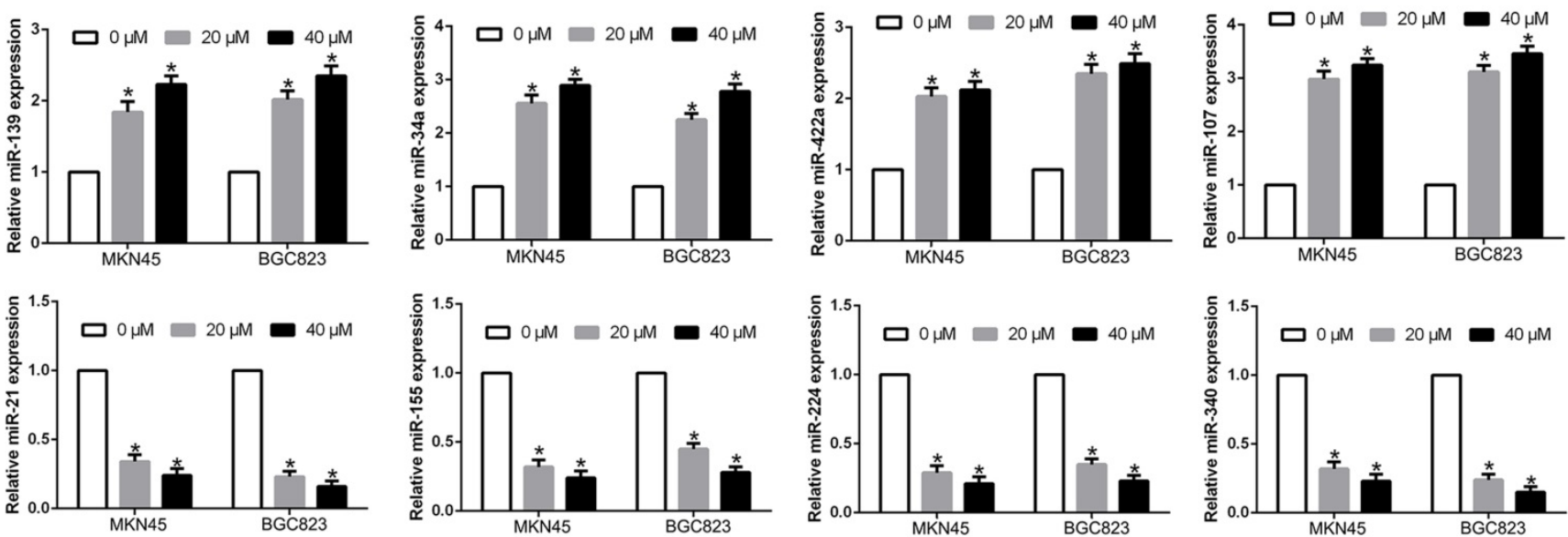

Figure 6. Luteolin treatment regulated the miRNAs expression levels. 


\section{Discussion}

Despite surgery resection and chemotherapy advancement in GC therapy, the long-term prognosis of advanced GC patients remains poor due to hepatic or distant lymph node metastasis and recurrence [30]. Recently, the chemical compound of natural extracts from plants has been reported to act as potential therapeutic agent for gastric cancer [31]. Luteolin, a novel dietary flavonoid from plants and foods, has been reported to inhibit the development and progression in different tumors [32]. Luteolin modulates cell cycle arrest and apoptosis in human colon cancer cells and xenografts. Luteolin reduces migration of human glioblastoma cell lines via inhibition of the p-IGF/PI3K/AKT/ mTOR signaling pathway [21]. Luteolin exerts an anticancer effect on NCI-H460 human non-small cell lung cancer cells through the induction of Sirt1-mediated apoptosis [32]. Therefore, we attempted to elucidate the biological effects and the underlying mechanisms of luteolin on GC.

In present study, we firstly confirmed that luteolin inhibited cell viability was time- and dose-dependent in GC cells. These results were consistent with previous studies in other cancers. Our results further demonstrated that luteolin suppressed cell cycle arrest, colony formation, proliferation and promoted apoptosis in GC cells in vitro and in vivo. These biological effects of luteolin on GC cells were mediated by regulating Cyclin D1, Cyclin E, p21, Bcl2 and Bax. These molecules were cycle and apoptosis-associated regulators. Moreover, most of cancer deaths are caused by metastasis. The metastasis of cancer is a complex processed that contains invasion and migration to the circulatory system. Our study evaluated the potential of luteolin effect on metastasis. We demonstrated that luteolin inhibited migration and invasion in vitro and suppressed the lung metastasis in vivo. Luteolin regulated metastasis through regulating MMPs expression and EMT process. Progress of invasion involves proteolytic degradation of the extracellular matrix by MMPs. Increasing studies indicated EMT, an evolutionarily conserved process in which cells undergo conversion of epithelial cells to mesenchymal cells, is recognized as the initiation of metastasis [33]. Our data suggest that luteolin regulated GC cell migration and invasion by MMPs and EMT. Moreover, to explore the molecular mechanisms of luteolin, we confirmed that luteolin regulated Notch1, PI3K, AKT, mTOR, ERK, STAT3 and P38 signaling pathway, which play a critical role in development and progression of GC. In addition, we confirmed that luteolin regulated a panel of miRNAs expression, which contribute to the biological function of GC.
These findings provide the first validated evidence suggesting that luteolin may be a novel and effective anticancer agent.

In conclusion, our results indicated that luteolin inhibited cell viability, induced cell cycle arrest, colony formation, proliferation, migration, invasion and promoted apoptosis in vitro and in vivo. We also illustrated that luteolin regulated multiple signaling pathways and miRNAs in GC cells. Our results promote further research into the use of luteolin in GC treatment.

\section{Abbreviations}

miRNAs, microRNAs; GC, gastric cancer; qRT-PCR, real-time quantitative reverse transcription polymerase chain reaction; IHC, immunohistochemistry; 3'-UTR: 3'-untranslated region; EMT: Epithelial-mesenchymal transition; H\&E: Hematoxylin and eosin.

\section{Acknowledgements}

This study was supported by scientific and technological project of X' $\mathrm{i}^{\prime}$ an (2017113SF/YX007(11)) and Shaanxi Provincal project of scientific and technological innovation team (2017KCT-28).

\section{Competing Interests}

The authors have declared that no competing interest exists.

\section{References}

1. Siegel R, Ma J, Zou Z, Jemal A. Cancer statistics, 2014. CA: a cancer journal for clinicians. 2014; 64: 9-29.

2. Torre LA, Bray F, Siegel RL, Ferlay J, Lortet-Tieulent J, Jemal A. Global cancer statistics, 2012. CA: a cancer journal for clinicians. 2015; 65: 87-108.

3. Chen W, Zheng R, Baade PD, Zhang S, Zeng H, Bray F, et al. Cancer statistics in China, 2015. CA: a cancer journal for clinicians. 2016; 66: 115-32.

4. Zong L, Abe M, Seto Y, Ji J. The challenge of screening for early gastric cancer in China. Lancet. 2016; 388: 2606.

5. Cervantes A, Roda D, Tarazona N, Rosello S, Perez-Fidalgo JA. Current questions for the treatment of advanced gastric cancer. Cancer treatment reviews. 2013; 39: 60-7.

6. Glockzin G, Piso P. Current status and future directions in gastric cancer with peritoneal dissemination. Surgical oncology clinics of North America. 2012; 21: 625-33.

7. Wang X, Ouyang Y, Liu J, Zhu M, Zhao G, Bao W, et al. Fruit and vegetable consumption and mortality from all causes, cardiovascular disease, and cancer: systematic review and dose-response meta-analysis of prospective cohort studies. Bmj. 2014; 349: g4490.

8. Nijveldt RJ, van Nood E, van Hoorn DE, Boelens PG, van Norren K, van Leeuwen PA. Flavonoids: a review of probable mechanisms of action and potential applications. The American journal of clinical nutrition. 2001; 74: 418-25.

9. Xiong J, Li S, Wang W, Hong Y, Tang K, Luo Q. Screening and identification of the antibacterial bioactive compounds from Lonicera japonica Thunb. leaves. Food chemistry. 2013; 138: 327-33.

10. $\mathrm{Xu} \mathrm{T}, \mathrm{Li} \mathrm{D}$, Jiang D. Targeting cell signaling and apoptotic pathways by luteolin: cardioprotective role in rat cardiomyocytes following ischemia/reperfusion. Nutrients. 2012; 4: 2008-19.

11. Lim DY, Jeong $\mathrm{Y}$, Tyner $\mathrm{AL}$, Park JH. Induction of cell cycle arrest and apoptosis in HT-29 human colon cancer cells by the dietary compound luteolin. American journal of physiology Gastrointestinal and liver physiology. 2007; 292: G66-75.

12. Seelinger G, Merfort I, Wolfle U, Schempp CM. Anti-carcinogenic effects of the flavonoid luteolin. Molecules. 2008; 13: 2628-51.

13. Lin D, Kuang G, Wan J, Zhang X, Li H, Gong X, et al. Luteolin suppresses the metastasis of triple-negative breast cancer by reversing 
epithelial-to-mesenchymal transition via downregulation of beta-catenin expression. Oncology reports. 2017; 37: 895-902.

14. Lee YJ, Lim T, Han MS, Lee SH, Baek SH, Nan HY, et al. Anticancer effect of luteolin is mediated by downregulation of TAM receptor tyrosine kinases, but not interleukin-8, in non-small cell lung cancer cells. Oncology reports. 2017; 37: 1219-26.

15. Han K, Meng W, Zhang JJ, Zhou Y, Wang YL, Su Y, et al. Luteolin inhibited proliferation and induced apoptosis of prostate cancer cells through miR-301. OncoTargets and therapy. 2016; 9: 3085-94.

16. Wang TT, Wang SK, Huang GL, Sun GJ. Luteolin induced-growth inhibition and apoptosis of human esophageal squamous carcinoma cell line Eca109 cells in vitro. Asian Pacific journal of cancer prevention : APJCP. 2012; 13: 5455-61.

17. Meng G, Chai K, Li X, Zhu Y, Huang W. Luteolin exerts pro-apoptotic effect and anti-migration effects on A549 lung adenocarcinoma cells through the activation of MEK/ERK signaling pathway. Chemico-biological interactions. 2016; 257: 26-34.

18. Cheng WY, Chiao MT, Liang YJ, Yang YC, Shen CC, Yang CY. Luteolin inhibits migration of human glioblastoma U-87 MG and T98G cells through downregulation of Cdc42 expression and PI3K/AKT activity. Molecular biology reports. 2013; 40: 5315-26.

19. Yang MY, Wang CJ, Chen NF, Ho WH, Lu FJ, Tseng TH. Luteolin enhances paclitaxel-induced apoptosis in human breast cancer MDA-MB-231 cells by blocking STAT3. Chemico-biological interactions. 2014; 213: 60-8.

20. Chen $\mathrm{P}$, Zhang JY, Sha BB, Ma YE, Hu T, Ma YC, et al. Luteolin inhibits cell proliferation and induces cell apoptosis via down-regulation of mitochondrial membrane potential in esophageal carcinoma cells EC1 and KYSE450. Oncotarget. 2017; 8: 27471-80.

21. Wang $\mathrm{Q}$, Wang $\mathrm{H}$, Jia $\mathrm{Y}$, Ding $\mathrm{H}$, Zhang $\mathrm{L}$, Pan $\mathrm{H}$. Luteolin reduces migration of human glioblastoma cell lines via inhibition of the p-IGF-1R/PI3K/AKT/mTOR signaling pathway. Oncology letters. 2017; 14: 3545-51.

22. Yu X, Ma C, Fu L, Dong J, Ying J. MicroRNA-139 inhibits the proliferation, migration and invasion of gastric cancer cells by directly targeting rho-associated protein kinase 1. Oncology letters. 2018; 15: 5977-82.

23. Deng X, Zheng H, Li D, Xue Y, Wang Q, Yan S, et al. MicroRNA-34a regulates proliferation and apoptosis of gastric cancer cells by targeting silent information regulator 1. Experimental and therapeutic medicine. 2018; 15: 3705-14.

24. He Z, Li Z, Zhang X, Yin K, Wang W, Xu Z, et al. MiR-422a regulates cellular metabolism and malignancy by targeting pyruvate dehydrogenase kinase 2 in gastric cancer. Cell death \& disease. 2018; 9: 505.

25. Cheng F, Yang Z, Huang F, Yin L, Yan G, Gong G. microRNA-107 inhibits gastric cancer cell proliferation and metastasis by targeting PI3K/AKT pathway. Microbial pathogenesis. 2018.

26. Li C, Song L, Zhang Z, Bai XX, Cui MF, Ma LJ. MicroRNA-21 promotes TGF-beta1-induced epithelial-mesenchymal transition in gastric cancer through up-regulating PTEN expression. Oncotarget. 2016; 7: 66989-7003.

27. Qu Y, Zhang H, Sun W, Han Y, Li S, Qu Y, et al. MicroRNA-155 promotes gastric cancer growth and invasion by negatively regulating transforming growth factor-beta receptor 2. Cancer science. 2018; 109: 618-28.

28. Zhang Y, Li CF, Ma LJ, Ding M, Zhang B. MicroRNA-224 aggrevates tumor growth and progression by targeting $\mathrm{mTOR}$ in gastric cancer. International journal of oncology. 2016; 49: 1068-80.

29. Yin G, Zhou H, Xue Y, Yao B, Zhao W. MicroRNA-340 promotes the tumor growth of human gastric cancer by inhibiting cyclin G2. Oncology reports. 2016; 36: 1111-8.

30. Cristescu R, Lee J, Nebozhyn M, Kim KM, Ting JC, Wong SS, et al. Molecular analysis of gastric cancer identifies subtypes associated with distinct clinical outcomes. Nature medicine. 2015; 21: 449-56.

31. Lu X, Li Y, Li X, Aisa HA. Luteolin induces apoptosis in vitro through suppressing the MAPK and PI3K signaling pathways in gastric cancer. Oncology letters. 2017; 14: 1993-2000.

32. Ma L, Peng H, Li K, Zhao R, Li L, Yu Y, et al. Luteolin exerts an anticancer effect on NCI-H460 human non-small cell lung cancer cells through the induction of Sirt1-mediated apoptosis. Molecular medicine reports. 2015; 12: 4196-202.

33. He Z, Dong W, Li Q, Qin C, Li Y. Sauchinone prevents TGF-beta-induced EMT and metastasis in gastric cancer cells. Biomedicine \& pharmacotherapy = Biomedecine \& pharmacotherapie. 2018; 101: 355-61. 